\title{
Thermo Mechanical Analysis of Polypropylene/Calcium Carbonate Nano Composite using Thermo Mechanical Analyzer
}

\author{
Prof. Tushar S Kulkarni ${ }^{1}$, Prof. Abhishek Kumar Jain ${ }^{2}$ \\ ${ }^{1}$ Department of Mechanical Engineering, Corporate Institute of Science and Technology, Bhopal, India \\ ${ }^{2}$ Department of Mechanical Engineering, Barkatullah University Institute of Technology, Bhopal, India
}

\begin{abstract}
Polypropylene is extensively used for varied applications due to its low cost, low density, high thermal stability and resistance to corrosion. Enhanced mechanical properties of Polypropylene can be achieved by mixing it with clays to form nanocomposites which increases its utility. Polypropylene Nano composites are formed by mixing polypropylene with calcium carbonate nano particles. This results in lighter materials with higher modulus and reduced linear thermal expansion making them desirable for some applications such as exterior automobile parts. The goal of this study is to determine thermo mechanical property ie Coefficient of Linear Thermal expansion of Polypropylene Calcium carbonate Nano composite using Thermo Mechanical Analyzer.
\end{abstract}

Keywords - Coefficient of linear thermal expansion, Polypropylene Nano composite, TMA, PNC, CTE.

\section{INTRODUCTION}

A Nanocomposite is as a multiphase solid material where one of the phases has one, two or three dimensions of less than 100 nanometers $(\mathrm{nm})$. They have been made from different sources and include the following types $\mathrm{Al}, \mathrm{FE}, \mathrm{Au}, \mathrm{Ag}, \mathrm{ZnO}, \mathrm{Al}_{2} \mathrm{O}_{3}, \mathrm{CaCO}_{3}, \mathrm{TiO}_{2}, \mathrm{SiO}_{2} \mathrm{SiC}$, Carbon black.

This definition can include porous media, colloids, gels and copolymers, but is more usually taken to mean the solid combination of a bulk matrix and nano-dimensional phase(s) differing in properties due to dissimilarities in structure and chemistry. The mechanical, electrical, thermal, optical, electrochemical, catalytic properties of the nanocomposite will differ from that of the component materials.

Size limits for these effects have been proposed, $<5 \mathrm{~nm}$ for catalytic activity, $<20 \mathrm{~nm}$ for making a hard magnetic material soft, $<50 \mathrm{~nm}$ for refractive index changes, and $<100 \mathrm{~nm}$ for achieving super para-magnetism, mechanical strengthening or restricting matrix dislocation movement.

Nano composites are found in nature, for example in the structure of the abalone shell and bone. The use of nano particle-rich materials long predates the understanding of the physical and chemical nature of these materials.

In mechanical terms, nanocomposites differ from conventional composite materials due to the exceptionally high surface to volume ratio of the reinforcing phase and/or its exceptionally high aspect ratio. The reinforcing material can be made up of particles (e.g. minerals), sheets (e.g. exfoliated clay stacks) or fibres (e.g. carbon nanotubes or electrospun fibres). The area of the interface between the matrix and reinforcement phase(s) is typically an order of magnitude greater than for conventional composite materials. The matrix material properties are significantly affected in the vicinity of the reinforcement. With polymer nano composites, properties related to local chemistry, degree of thermoset cure, polymer chain mobility, polymer chain conformation, degree of polymer chain ordering or crystallinity can all vary significantly and continuously from the interface with the reinforcement into the bulk of the matrix.

This large amount of reinforcement surface area means that a relatively small amount of nanoscale reinforcement can have an observable effect on the macroscale properties of the composite. For example, adding carbon nanotubes improves the electrical and thermal conductivity.

Other kinds of nano particulates may result in enhanced optical properties, dielectric properties, heat resistance or mechanical properties such as stiffness, strength and resistance to wear and damage. Polymer and polymer composite materials offer a wide range of advantages such as high strength-to-weight ratio, impact resistance, high flexibility, recyclability, corrosion resistance, low cost, and fast processing time, which make them very attractive materials. 


\section{POLYPROPYLENE CALCIUM CARBONATE NANOCOMPOSITES}

Polymer nanocomposites (PNCs) are significant technological advancement in the plastics industry. PNCs are formed from blends of nanometer sized fillers with either thermoplastics or thermosetting polymers. PNCs improves mechanical properties, barrier properties heat resistance, dimensional stability and flame retardancy.

There are many types of nano sized fillers being used among which include nano fibres such as carbon nanotubes or cellulose whiskers, nano platelets such as layered silicate and nano-sized isotropic particles such as silica or calcium carbonate $\left(\mathrm{CaCO}_{3}\right)$.

$\mathrm{CaCO}_{3}$ is one of the most commonly used fillers in thermoplastics, especially polyolefins . It is inexpensive and can be used at high loadings. Earlier it was used as a cheap extender, fine grades of precipitated $\mathrm{CaCO}_{3}$ is now being produced in nanometer size and this nano precipitated $\mathrm{CaCO}_{3}$ (NPCC) has the potential to be an important functional filler in polypropylene composites.

The evaluation of filler dispersion is important as only well dispersed nanoparticles can lead to the expected improvement in mechanical properties. For the nanocomposites to possess good impact toughness, it is critical that the filler dispersion in the polymer matrix be very uniform to avoid creation of crack-initiating large agglomerates.

\section{THERMO MECHANICAL ANALYSIS (TMA) :}

Is a technique used in thermal analysis, a branch of materials science which studies the properties of materials as they change with temperature. Thermo mechanical analysis is a subdiscipline of the thermo mechanometry (TM) technique. Thermo mechanometry is the measurement of a change of a dimension or a mechanical property of the sample while it is subjected to a temperature regime. An associated thermo analytical method is thermo mechanical analysis. Thermo dilatometry is similar technique for the measurement of a change of a dimension of the sample with a negligible force acting on the sample while it is subjected to a temperature regime.

Thermo-mechanical analysis (TMA) is one of the important characterization techniques in the field of thermal analysis. With TMA, the dimensional properties of a sample are measured as the sample is heated, cooled or held under isothermal conditions. The loading or force applied to the sample can be varied with TMA. The technique is used to assess the following important properties of polymers :

- Softening temperatures or glass transition temperature $\operatorname{Tg}(\mathrm{s})$

- Melting temperatures

- Stress relief effects at $\mathrm{Tg}$

- Coefficients of thermal expansion (CTE)

- Dimensional compatibilities of two or more different materials

- Onset of foaming

- Relative degree of cure of thermosets

- Composite delamination temperatures

- Percent shrinkages of films and fibers

- Shrinkage forces

- Effectiveness of cling of films

- Testing of coatings on metals, films, optical fibers and electrical wires

- Assessment of transverse versus machine orientational properties of films.

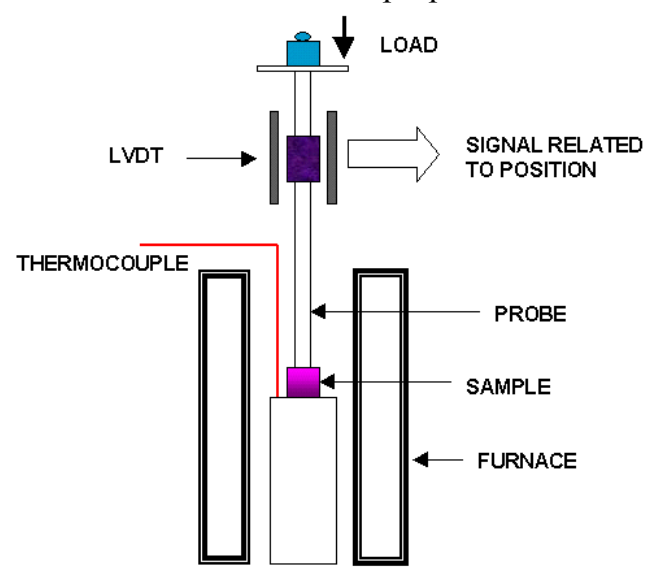

Thermo Mechanical Analyzer 
Different probe configurations are offered to perform TMA in order to optimize the test conditions for a specific sample and/or application. The most commonly used TMA probe is the expansion probe. This probe rests on the surface of the test specimen under low loading conditions. As the sample expands, during heating, the probe is pushed up and the resulting expansion of the sample is measured.

Coefficients of thermal expansion (CTE) can be easily measured by TMA. The CTE is a quantitative assessment of the expansion of a material over a temperature interval. When manufacturing products that contain two different materials, it is oftentimes critical to ensure that the materials will have CTE values that are identical to avoid the build-up of thermal stresses or to prevent leaks or component malfunctions.

\subsection{Linear Coefficient of Thermal Expansion :}

When heat is added to most materials, the average amplitude of the atoms vibrating within the material increases. This, in turn, increases the separation between the atoms causing the material to expand. If the material does not go through a phase change, the expansion can be easily related to the temperature change. The linear coefficient of thermal expansion describes the relative change in length of a material per degree temperature change.

If the linear coefficient of thermal expansion is known, the change in components length can be calculated for each degree of temperature change. This effect also works in reverse. That is to say, if energy is removed from a material then the object's temperature will decrease causing the object to contract.

Thermal expansion (and contraction) must be taken into account when designing products with close tolerance fits as these tolerances will change as temperature changes if the materials used in the design have different coefficients of thermal expansion. It should also be understood that thermal expansion can cause significant stress in a component if the design does not allow for expansion and contraction of components. The phenomena of thermal expansion can challenging when designing bridges, buildings, aircraft and spacecraft, but it can be put to beneficial uses. For example, thermostats and other heat-sensitive sensors make use of the property of linear expansion.

\subsection{Significance and Use :}

The coefficient of linear thermal expansion (CLTE), $\alpha$, between temperatures $T_{1}$ and $T_{2}$ for a specimen whose length is $\mathrm{L}_{0}$ at the reference temperature, is given by the following equation:

$\alpha=\left(\mathrm{L}_{2}-\mathrm{L}_{1}\right) /\left\{\mathrm{L}_{\mathrm{o}}\left(\mathrm{T}_{2}-\mathrm{T}_{1}\right)\right\}=\Delta \mathrm{L} / \mathrm{L}_{\mathrm{o}} \Delta \mathrm{T}$

where $L_{1}$ and $L_{2}$ are the specimen lengths at temperatures $T_{1}$ and $T_{2}$, respectively. $\alpha$ is, therefore, obtained by dividing the linear expansion per unit length by the change in temperature.

Any material expands when heated. The Coefficient of Linear Thermal Expansion (CLTE) is used to calculate changes in length, breadth and thickness of a moulded part with changes in temperature. Addition of fillers, such as glass, significantly alters the CLTE of a polymer. Polymers may be formulated with CLTEs to match those of metal or other materials used in complex components, such as automotive parts. With test method ASTM E631 CLTE is measured using a thermomechanical analyser. The sample is placed in an enclosure and is in contact with a probe leading to a displacement sensor. A small force is applied to keep the probe in contact with the sample. The temperature within the enclosure is raised at a rate of $5^{\circ} \mathrm{C}$ per minute and the expansion of the sample is measured by the displacement sensor. ASTM E631 covers temperatures between $-120^{\circ} \mathrm{C}$ and $+600^{\circ} \mathrm{C}$.

\section{IV.EXPERIMENTAL}

To evaluate Co-efficient of Linear Thermal Expansion (CLTE) of polypropelene calcium carbonatge (5\%) nano composite.

\subsection{Methodology:}

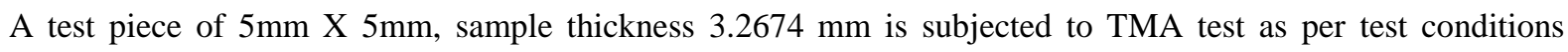
below. The obtained TMA plot is evaluated for the coefficient of linear thermal expansion in the temperature range from the ambient to the maximum temperature after which the material shows softening.

\subsection{Test Conditions:}

a) Equipment: Thermo Mechanical Analyser TMA 40, Mettler Toledo make.

b) Method: Dynamic TMA, as tabulated below :- 
Conditions :

Observation table :

\begin{tabular}{|c|c|c|c|}
\hline Test Description & $\begin{array}{c}\text { TMA } \\
\text { Plot no. 01 }\end{array}$ & Temperature range & Observed value \\
\hline CLTE & 1 & $40-152.24$ & $205.07 \times 10-6$ \\
$\mu \mathrm{m} / \mathrm{meter} /{ }^{\circ} \mathrm{C}$
\end{tabular}

\section{3 Graphs and figures :}

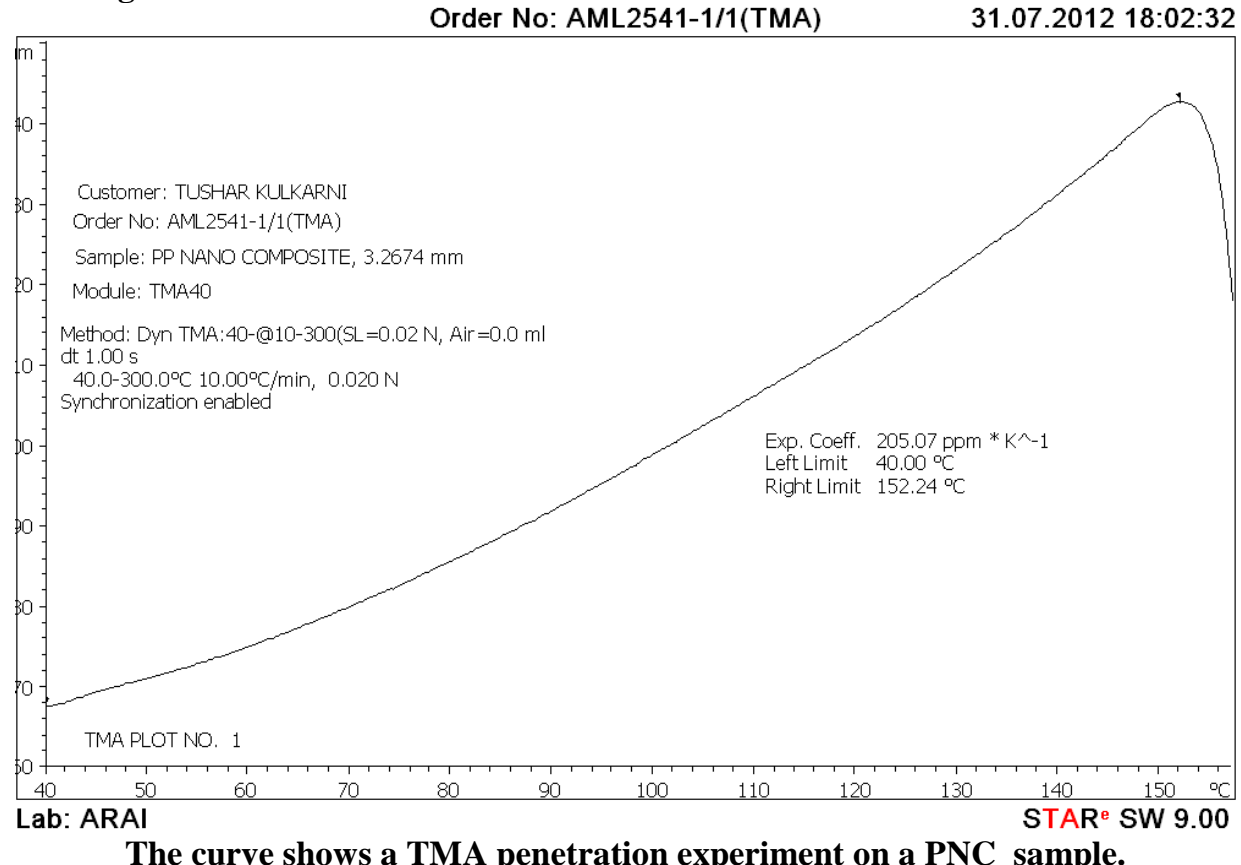

\section{Experimentation procedure :-}

1). A sample of Nano composite containing $95 \%$ polypropylene and $5 \%$ calcium carbonate having a dimensions of $5 \mathrm{~mm} \mathrm{X} 5 \mathrm{~mm} \times 3.2674 \mathrm{~mm}$ is clamped on the fixture on the Thermo mechanical analyzer.

2). The Temperature on the analyzer is set at room temperature $40{ }^{\circ} \mathrm{C}$. Rate of Heating ${ }^{\circ} \mathrm{C} / \mathrm{min}$ is $10^{\circ} \mathrm{C}$.

3). The penetration probe is set on the mid of the sample which is geometric centre of the square piece.

4). A constant load of $0.02 \mathrm{~N}$ is set to be applied on the probe.

5). Temperature is changed to $50,60,70,80,90,100,110,120,130,140,150$ Degree $\mathrm{C}$ per minute respectively. The change in the dimension to corresponding temperature observed and recorded.

6). The sample due to the influence of the heat and the applied force on the penetration probe sags uniformly without loosing its sectional thickness.

7). At approximately $150{ }^{\circ} \mathrm{C}$ the sample starts loosing its total rigidity as it reaches to melting point, the probe movement remains stable and at $145 \mu \mathrm{m}$ it starts moving up for a while as the sample totally looses its shape changing to molten state.

8). The auto shut stops the analyzer as safety measure to avoid the damage to quartz tip on probe.

9). The Analyzer while the experiment is connected to a computer which is programmed to capture the reading through an analysis software which plots the graph and calculates the mean value for coefficient of thermal expansion using the displacement $\mathrm{v} / \mathrm{s}$ temperature data obtained by the analyzer.

\section{OBSERVATIONS}

1). Using a penetration probe, the sample with thickness $3.2674 \mathrm{~mm}$ was scanned from room temperature from 40.0 up to $152.24^{\circ} \mathrm{C}$ with a force of $0.020 \mathrm{~N}$. The sample softens above $152.24{ }^{\circ} \mathrm{C}$.

2). The thermal curve is generated from the actual experimentation values with ordinate taken as displacement ranging from 60 to $145 \mu \mathrm{m}$ and temperature on abscissa.

3). The thermal curve shows behavior of the nano composite while it is under the influence of gradual heat temperature 40 to $150^{\circ} \mathrm{C}$ The probe starts deflecting as the temperature raises to $40{ }^{\circ} \mathrm{C}$ from $60-$ $145 \mu \mathrm{m}$. The observations shows the nano composite maintains a uniform curve through out the temperature conditions. 
4). The Coefficient of Linear Thermal Expansion (CLTE) in thickness direction is 205.07 parts per million ( micrometer per meter) $/{ }^{\circ} \mathrm{C}$ in the temperature range of $40.0-152.24 \mathrm{Deg} \mathrm{C}$ as calculated by the analyzer.

\section{FUTURE SCOPE}

Polymer composites are the engineering materials. Development of nano composites has given a new direction to fulfil the need of better material for the application in Defence, Automobiles, electronics, machines pertaining to light weight, low cost, environmental friendly material.

The CLTE of PPCCNC is one of the property which has been focused to analyze a nano composite for the development efficient and better material for the engineering applications. Thermomechanical analyzer equipment facilitates accurate and precise values for the variation of length due to effect of temperature conditions. There fore ensuring the capability, functio ability, durability and safety of the part. The TMA analysis is made at micro level, the readings and observations are obtained very accurately.

\section{ACKNOWLEDGEMENT}

Sample of Polypropelene calcium carbonate nano composite developed provided by Dr Ajay Kumar Nema

The test has been performed at Automotive Research association of India, Pune on Thermo Mechanical Analyzer Metler Toledo TMA 40 make. Test Report no AML/2012-07/2541/239 Dated 23/08/2012 OC no. 2541.

\section{REFERENCES}

1). Structural and mechanical properties of polymer nanocomposites S.C. Tjong Materials Science and Engineering R 53 (2006) 73-197.

2). Can nanoparticles really enhance thermal stability of polymers : An overview on thermal decomposition of addition polymers K.Chrissafis, D. Bikiaris Thermochimica Acta 523 (2011) 1- 24

3). Polypropylene/calcium carbonate nanocomposites Chi Ming Chan, Jing shen Wu, Jian Xiong Li, Ying Kit Cheung. Polymer 43 (2002) 2981-2992.

4). The behaviour features of polymer nanocomposites filled with calcium carbonate. Georgiy Kozlov, Zarema Aphashagova, Akhmed Malamatov and Gennady Zaikov. Chem and chem. Technolgy Vol. 6, No. 1, 2012.

5). Defence Applications of Polymer Nanocomposite R.V. Kurahatti, A.O. Surendranathan, S. A. Kori, Nirbhay Singh. A.V. Ramesh Kumar., and Saurabh Srivastava. Defence Science Journal, Vol. 60, No. 5, September 2010, pp. 551-563 Ó 2010, DESIDOC.

6). Polymeric Nanocomposites for Automotive Applications By Juan M. Garcos, David J. Moll, Jozef Bicerano, Richard Fibiger, and David G. McLeod Adv. Mater. 2000, 12, No. 23, December 1.

7). Oxygen barrier property of polypropylene-polyether treated clay nanocomposite $M$. Pannirselvam, A. Genovese, M. C. Jollands, S. N. Bhattacharya, R. A. Shank. eXPRESS Polymer Letters Vol.2, No.6 (2008) 429-439.

8). Polypropylene/calcium carbonate nanocomposites - effects of processing techniques and maleated polypropylene compatibiliser M. Y. A. Fuad, H. Hanim1, R. Zarina, Z. A. Mohd. Ishak, Azman Hassan. Express Polymer Letters Vol.4, No.10 (2010) 611-620.

9). Clay containing polymer nanocomposite Technology :; The staple for modern plastics industry .Suprakas Sinha Ray DST/CSIR Nanotechnology Innovation Centre, National Centre for NanoStructured Materials, Council for Scientific and Industrial Research, Pretoria 001, South Africa.

10). Effect of mixer type on exfoliation of polypropylene nanocomposites. Michail K. Dolgovskija, Paula D. Fasulob, Frédéric Lortiea, Christopher W. Macoskoa, Robert A. Ottavianib, William R. Rodgersb ANTEC 2003 / 2259.

11). Litreture survey of current state of current state of research on nanocomposites and Multifunctional materials. Christopher C. Ibeh, David Hui, Kunigal Shivakumar.

12). Thermal and Mechanical Properties of Modified CaCO /PP Nanocomposites A. Buasri, N. Chaiyut, K. Borvornchettanuwat, N. Chantanachai and K. Thonglor World Academy of Science, Engineering and Technology 682012.

13). Polypropylene Compounds for Automotive Applications Satoru Moritomi, Tsuyoshi Watanbe, Susumu Kanzaki.

14). Polymer nanotechnology: Nanocomposites D.R. Paul , L.M. Robeson Polymer 49 (2008) 31873204.

15). Mechanical Properties of Polypropylene/Calcium Carbonate Nanocomposites Daniel Eirasa, 
Luiz Antonio Pessan, Materials Research, Vol. 12, No. 4, 517-522, 2009.

16). Modulated Thermomechanical Analysis - Measuring Expansion and Contraction Simultaneously Roger L. Blaine, Ph.D TA Systems US.

17). Thermal Expansions ASM Ready Reference: Thermal Properties of Metals ASM International, Materials Park, Ohio, USA 06702G.

18). Physicomechanical study of random polypropylene filled with treated and untreated nanocalcium carbonate: Effect of different coupling agents and compatibilizer Pradeep Upadhyaya, Ajay K. Nema jtc.sagepub.com/content/early/2012/03/05/0892705711433349.

19). Polymer Nanocomposites Arunkumar Lagashetty and A Venkataraman.

20). Thermal Conductivity of Nanoparticles Filled Polymers Hassan Ebadi-Dehaghani and Monireh Nazempour Shahreza Branch, Islamic Azad University Iran.

21). Clay on Mechanical, Thermal and Morphological Properties of Ethylene Octane Copolymer Polypropylene Nanocomposites .S. K. Sharma, Ajay K. Nema and S. K. Nayak. 